\title{
Can Typicality Arguments Dissolve Cosmology's Flatness Problem?
}

\author{
C.D. $\mathrm{McCoy}^{*}$
}

20 February 2016

\begin{abstract}
The flatness problem in cosmology draws attention to a surprising fine-tuning of the spatial geometry of our universe towards flatness. Several physicists, among them Hawking, Page, Coule, and Carroll, have argued against the probabilistic intuitions underlying such fine-tuning arguments in cosmology and instead propose that the canonical measure on the phase space of Friedman-Robertson-Walker spacetimes should be used to evaluate fine-tuning. They claim that flat spacetimes in this set are actually typical on this natural measure and that therefore the flatness problem is illusory. I argue that they misinterpret typicality in this phase space and, moreover, that no conclusion can be drawn at all about the flatness problem by using the canonical measure alone.
\end{abstract}

For several decades now cosmologists have maintained that the old standard model of cosmology, the highly successful hot big bang (HBB) model, suffers from various fine-tuning problems (Dicke and Peebles, 1979. Linde, 1984). They claim that the spacetimes on which the HBB model is based, the Friedman-RobertsonWalker (FRW) spacetimes, require seemingly "special" initial conditions, such that when they are evolved forward in time by the dynamical law of the general theory of relativity (GTR) they yield presently observed cosmological conditions. For example, the flatness problem depends on the existence of special initial conditions in the HBB model which are required to explain the observationally-inferred spatial flatness of the universe. Due to their extreme precision or intuitive "unlikeliness," these initial conditions are thought to be unduly special, such that many cosmologists have felt that the initial conditions themselves are in need of explanation and, moreover, present a significant conceptual problem for the HBB model.

Although physical fine-tuning could be interpreted in a variety of ways, cosmologists typically understand it to mean that observationally-required initial conditions are in some sense unlikely (Smeenk, 2013, McCoy, 2015). In order to substantiate this interpretation, one must show that initial conditions in the HBB model which reproduce present conditions are in fact unlikely. This task presupposes that there is a justifiable way of assessing the likelihoods of cosmological models (Gibbons et al., 1987; Hawking and Page, 1988). Many arguments found in the cosmological literature, however, rely on ad hoc, unjustified likelihood measures. Gibbons et al. (1987) propose a "natural" measure (hence the GHS measure) on the set of FRW spacetimes (with matter contents represented by a scalar field) as a natural and justified way of evaluating likelihoods. The GHS measure is simply the canonical Liouville measure associated with the phase space of FRW spacetimes when GTR is put into a Hamiltonian formulation and in a precise sense "comes for free" with the phase space.

While I would maintain that the GHS measure cannot be successfully used to make arguments about finetuning in cosmology quite generally, I argue here only for its inapplicability to the flatness problem. Some

*Eidyn Research Centre, University of Edinburgh, Edinburgh, UK. email: casey .mccoy@ed.ac.uk 
authors (Gibbons and Turok, 2008; Carroll and Tam, 2010) have attempted to make probabilistic arguments, in analogy to familiar probabilistic arguments in statistical mechanics, by making the GHS measure into a probability measure. However, as the total measure of the FRW phase space is infinite, there is no canonical choice of probability measure with which to make probabilistic arguments, a point that has been recognized already by some (Hawking and Page, 1988, Schiffrin and Wald, 2012). Accordingly, any justification of a particular probability measure is completely independent of the justification of the GHS measure-in short, these probability measures are not in any substantive sense the GHS measure. On the other hand, one might try to use the GHS measure by itself to make typicality arguments in analogy to typicality arguments in statistical mechanics (Goldstein, 2012). Carroll in particular advocates this approach and, interestingly, claims that the GHS measure alone tells us that almost all spacetimes are spatially flat (Carroll and Tam. 2010, Remmen and Carroll, 2013, Carroll, forthcoming) - that there is in fact no flatness problem (Hawking and Page (1988, 803-4) and Coule (1995, 468) suggest the same). Carroll's claim, however, rests on a subtle mistake in interpreting typicality. I claim, on the contrary, that the GHS measure cannot tell us anything about likelihood without substantive additional assumptions such as those made in statistical mechanics, e.g. a partition of phase space into "macroproperties" or similar. These necessary assumptions, however, are doubtfully justifiable in the cosmological context. Thus I ultimately conclude that the GHS measure cannot be used to clarify the nature of fine-tuning in cosmology.

\section{The Gibbons-Hawking-Stewart Measure}

An adequate view of what the GHS measure is and can do relies on understanding the details of how it is introduced. For this reason I develop here the measure with considerably more care than other accounts in the literature, which tend to jump straight to a Lagrangian or Hamiltonian formulation of GTR without elucidating the geometrical origin of their variable choices and the relations between physical parameters.

My starting point is the initial value formulation of GTR, in which the "position" initial data of spacetime are represented by the spatial metric $h_{a b}$ on a spacelike Cauchy surface $\Sigma$ and the "momentum" initial data by the extrinsic curvature $\pi_{a b}$ (Wald, 1984, Malament, 2012). FRW spacetimes are spacetimes with homogeneous and isotropic spacelike hypersurfaces, so one can foliate the spacetimes by a one-parameter family of these spacelike hypersurfaces $\Sigma_{t}$ that are orthogonal to a smooth, future-directed, twist-free, unit timelike field $\xi^{a}$ on $M$, where I define $\xi^{a}=\nabla^{a} t$.For FRW spacetimes the extrinsic curvature of an initial data surface $\Sigma_{t}$ is $H h_{a b}$, where $H$ is the so-called Hubble parameter.Thus the initial data for an FRW spacetime are completely represented by two objects: (1) the spatial metric $h_{a b}$ and (2) the Hubble parameter $H$ associated with a spatial hypersurface $\Sigma$.

The space of initial data is therefore the product of the set of homogeneous and isotropic Riemannian manifolds $\Sigma$ (with metric $h_{a b}$ ) and the set of (real-valued) Hubble parameters $H$. Homogeneous and isotropic Riemannian manifolds have constant curvature $\kappa$. Complete, connected Riemannian manifolds of constant sectional curvature are called space forms. It is a theorem that every simply-connected three-dimensional space form is isometric to the sphere $S^{3}(\sqrt{(}(1 / \kappa))$ if $\kappa>0, \mathbf{R}^{3}$ if $\kappa=0$, or the hyperbolic space $H^{3}(\sqrt{(}(1 / \kappa))$ if $\kappa<0$ (Wolf, 2010). The standard metrics on each of these manifolds is understood to be the metric induced on them by embedding them in $\mathbf{R}^{4}$. Every $\Sigma$ is therefore isometric to one of these three classes of space forms. Spaceforms of each of the three kinds are moreover homothetic, i.e. they are isometric up to the square of a scale factor $a$ (McCabe, 2004). Accordingly one has the means to represent curvature $\kappa$ as a function of the scale factor; in particular, for any $\Sigma, a^{2} \kappa$ is some constant $k$. Hence one can set any spatial metric $h_{a b}=a^{2} \gamma_{a b}$, where $\gamma_{a b}$ is the standard metric on the appropriate space form. This is useful in the initial value formulation of FRW spacetimes because all time dependence of $h_{a b}$ is thereby located solely in 
the scale factor rather than in the radius of curvature of the space form.

The Einstein equation reduces to two constraint equations and two evolution equations in the initial value formulation (Geroch, 1972):

$$
\begin{gathered}
\mathcal{R}-\left(\pi_{a}{ }^{a}\right)^{2}+\pi_{a b} \pi^{a b}=-16 \pi T_{a b} \xi^{a} \xi^{b} ; \\
D_{c} \pi_{a}^{c}-D_{a} \pi_{c}{ }^{c}=8 \pi T_{m r} h^{m}{ }_{a} \xi^{r} ; \\
£_{\xi}\left(\pi_{a b}\right)=2 \pi_{a}{ }^{c} \pi_{c b}-\pi^{c}{ }_{c} \pi_{a b}+\mathcal{R}_{a b}-8 \pi h_{a}{ }^{m} h^{n}{ }_{b}\left(T_{m n}-\frac{1}{2} T h_{m n}\right) ; \\
\mathfrak{\xi}_{\xi}\left(h_{a b}\right)=2 \pi_{a b},
\end{gathered}
$$

where $\mathcal{R}$ is the Ricci scalar of $\Sigma, \mathcal{R}_{a b}$ is the Ricci tensor of $\Sigma$, and $D_{a}$ is the derivative operator on $\Sigma$. For FRW spacetimes, these equations simplify to the following three (the second equation from above is trivial since $\pi_{a b}$ does not vary across $\Sigma$ :

$$
\begin{gathered}
\mathcal{R}-6 H^{2}=-16 \pi \rho ; \\
\dot{H} h_{a b}=\left(-H^{2}-\frac{4 \pi}{3}(\rho+3 p)\right) h_{a b} ; \\
\dot{h}_{a b}=2 H h_{a b},
\end{gathered}
$$

where $\rho$ is the energy density and $p$ the pressure of the matter. The first two equations are known as the Friedman equations. Since $h_{a b}=a^{2} \gamma_{a b}, \dot{h}_{a b}=2 a \dot{a} \gamma_{a b}$, and $2 H h_{a b}=2 H a^{2} \gamma_{a b}$, it follows from the third equation above that

$$
H=\frac{\dot{a}}{a}
$$

which is the usual definition of the Hubble parameter $H$. To simplify matters somewhat and to make contact with the literature, I shall henceforth take the matter contents of spacetime to be a scalar field $\phi$ in a potential $V$ which evolves according to the coupled Einstein-Klein Gordon equation 1 Then one has the following equations of motion (Hawking and Page, 1988, 790):

$$
\begin{gathered}
\mathcal{R}-6 H^{2}=-16 \pi\left(\frac{1}{2} \dot{\phi}^{2}+V(\phi)\right) \\
\dot{H}=-H^{2}-\frac{8 \pi}{3}\left(\frac{1}{2} \dot{\phi}^{2}-V(\phi)\right) \\
\ddot{\phi}+3 H \dot{\phi}+V^{\prime}(\phi)=0,
\end{gathered}
$$

where $V^{\prime}$ is the derivative of the potential with respect to $\phi$. (The third equation can be derived from the previous two, and so is in fact redundant.)

For FRW spacetimes the spatial Ricci scalar is $\mathcal{R}=-6 \kappa$. As noted before, one can cast $\kappa$ in terms of the scale factor and a constant $k: \kappa=k / a^{2}$. By using the scale factor $a$ to replace $\kappa$, one has introduced a constant $k$ which has no physical significance beyond identifying whether the space form is flat, positively-curved, or negatively-curved. One therefore usually takes equivalence classes of curves according to these three cases and chooses $k=+1,0$, and -1 as representatives. Then one may write $\mathcal{R}=-6 k / a^{2}$, so that one finally has Friedman's equation in its usual form (for a scalar field in a potential):

$$
\left(\frac{\dot{a}}{a}\right)^{2}=\frac{8 \pi}{3}\left(\frac{1}{2} \dot{\phi}^{2}+V(\phi)\right)-\frac{k}{a^{2}} .
$$

\footnotetext{
${ }^{1}$ The scalar field is meant to be the inflaton, the field that drives inflation in the early universe.

${ }^{2}$ If our interest were solely in assessing the HBB model's fine-tuning, one could do the following analysis for perfect fluid matter contents. The results would be qualitatively similar however, as shown by Carroll and Tam $[2010$ §4.2).
} 
The foregoing indicates that our FRW initial data $h_{a b}$ and $\pi_{a b}$ are equivalently representable in the space $\{a, \dot{a}, \phi, \dot{\phi}, k\}$. This space is not the space of initial data, however, since the previous equation is a constraint that must be satisfied by initial data. One must also keep in mind that $k$ is an index for three separate copies of the space $\{a, \dot{a}, \phi, \dot{\phi}\}$. There is no continuous path between the three spaces.

Have identified the relevant spaces for representing FRW space forms, I next put the theory into a Hamiltonian formulation (Wald 1984. Appendix E) in order to obtain a symplectic structure and, hence, the canonical measure. I begin with the Lagrangian for our theory of FRW spacetimes with a scalar field as the matter contents, where I have re-introduced the lapse function $N$ as a Lagrange multiplier:

$$
\mathcal{L}=\sqrt{-g}\left(\frac{R}{16 \pi}+\frac{1}{2 N^{2}} \dot{\phi}^{2}-V(\phi)\right) .
$$

In terms of the variables I have chosen, this is

$$
\mathcal{L}=-\frac{1}{8 \pi}\left(\frac{3}{N} a \dot{a}^{2}-3 N a^{3} \frac{k}{a^{2}}\right)+\frac{1}{2 N} a^{3} \dot{\phi}^{2}-N a^{3} V(\phi),
$$

in agreement with (Hawking and Page, 1988, Gibbons and Turok, 2008, Carroll and Tam, 2010). The momenta of $a$ and $\phi$ are

$$
p_{a} \equiv \frac{\partial \mathcal{L}}{\partial \dot{a}}=\frac{-3 a \dot{a}}{4 \pi N} ; \quad \quad p_{\phi} \equiv \frac{\partial \mathcal{L}}{\partial \phi}=\frac{a^{3} \dot{\phi}}{N} .
$$

The Hamiltonian on this phase space is

$$
\mathcal{H}=p_{a} \dot{a}+p_{\phi} \dot{\phi}-\mathcal{L}=N\left(-\frac{2 \pi p_{a}^{2}}{3 a}+\frac{p_{\phi}^{2}}{2 a^{3}}+a^{3} V(\phi)-a^{3} \frac{3}{8 \pi} \frac{k}{a^{2}}\right),
$$

from which one recovers (after setting $N=1$ ) our constraint (the Friedman equation) as the Hamiltonian constraint $C$ :

$$
C \equiv-\frac{2 \pi p_{a}^{2}}{3 a}+\frac{p_{\phi}^{2}}{2 a^{3}}+a^{3} V(\phi)-a^{3} \frac{3}{8 \pi} \frac{k}{a^{2}}=0 .
$$

The phase space $\gamma$ of our system is thus the four-dimensional space $\left\{a, p_{a}, \phi, p_{\phi}\right\}$ equipped with the canonical symplectic form

$$
\omega_{p_{a}, a, p_{\phi}, \phi}=\mathrm{d} p_{a} \wedge \mathrm{d} a+\mathrm{d} p_{\phi} \wedge \mathrm{d} \phi
$$

The dynamically accessible phase space points are constrained to be on the three-dimensional hypersurface $C$. Thus it would be inappropriate to use $\omega$ for constructing a canonical volume measure on phase space. One can, however, pull the symplectic form back onto the constraint surface by first solving the constraint for $p_{\phi} !^{3}$

$$
p_{\phi}=a^{3}\left(\frac{4 \pi}{3} \frac{p_{a}^{2}}{a^{4}}+\frac{3}{4 \pi} \frac{k}{a^{2}}-2 V(\phi)\right)^{1 / 2} .
$$

Following Carroll and Tam, I also switch coordinates from $p_{a}$ to $H$, so that

$$
p_{\phi}=a^{3}\left(\frac{3}{4 \pi}\left(H^{2}+k / a^{2}\right)-2 V(\phi)\right)^{1 / 2}
$$

\footnotetext{
${ }^{3}$ The scalar field can have positive or negative momentum, so strictly speaking there should be $\mathrm{a} \pm$ in the following equation. The reader is welcome to annotate the equations that follow.
} 
and

$$
\mathrm{d} p_{a}=-\frac{3}{4 \pi}\left(2 a H \mathrm{~d} a+a^{2} \mathrm{~d} H\right)
$$

The differential of $p_{\phi}$ is then

$$
\mathrm{d} p_{\phi}=\frac{(3 / 4 \pi) a^{3} H \mathrm{~d} H-a^{3} V^{\prime} \mathrm{d} \phi+6 a^{2}\left(\left(3 H^{2}+2 k / a^{2}\right) / 8 \pi-V\right) \mathrm{d} a}{\left((3 / 4 \pi)\left(H^{2}+k / a^{2}\right)-2 V\right)^{1 / 2}} .
$$

Substituting these into $\omega$ then gives the pullback of the symplectic form onto $C$. The result is the following (pre-symplectic) differential form:

$$
\omega_{a, H, \phi}=\Theta_{H a}(\mathrm{~d} H \wedge \mathrm{d} a)+\Theta_{H \phi}(\mathrm{d} H \wedge \mathrm{d} \phi)+\Theta_{a \phi}(\mathrm{d} a \wedge \mathrm{d} \phi),
$$

where

$$
\begin{gathered}
\Theta_{H a}=-\frac{3}{4 \pi} a^{2} ; \\
\Theta_{H \phi}=\frac{(3 / 4 \pi) a^{3} H}{\left((3 / 4 \pi)\left(H^{2}+k / a^{2}\right)-2 V\right)^{1 / 2}} ; \\
\Theta_{a \phi}=\frac{6 a^{2}\left(\left(3 H^{2}+2 k / a^{2}\right) / 8 \pi-V\right)}{\left((3 / 4 \pi)\left(H^{2}+k / a^{2}\right)-2 V\right)^{1 / 2}} .
\end{gathered}
$$

This form is not symplectic (it is degenerate), so one cannot construct a natural volume measure on $C$. Ideally, the "real" phase space of our system would be given by "solving the dynamics," and then taking equivalence classes of phase points that are part of the same trajectory. In this way one would obtain the space of motions, onto which one could then pull back the degenerate form to obtain a new symplectic form (of degree two less than $\omega$ ) and construct a canonical measure. This is quite complicated in general due to the differential equation that must be solved. The usual approach to take instead is to set $H$ to some value $H_{*}$ in the differential form and define their measure accordingly, i.e. set

$$
\mathrm{d} \Omega=\left.\omega_{a, H, \phi}\right|_{H=H_{*}}=\left.\Theta_{a \phi}\right|_{H=H_{*}} \mathrm{~d} a \mathrm{~d} \phi .
$$

One may do this because surfaces of constant Hubble parameter in phase space are transverse to temporal evolution, and the measure is preserved under translation of these surfaces along the Hamiltonian flow. Finally, one may naturally define the GHS measure $\mu_{G H S}$ on Lebesgue measurable sets $U$ by

$$
U \mapsto \int_{U} \mathrm{~d} \Omega=-6 \int_{U} a^{2} \frac{\left(3 H_{*}^{2}+2 k / a^{2}\right) / 8 \pi-V}{\left((3 / 4 \pi)\left(H_{*}^{2}+k / a^{2}\right)-2 V\right)^{1 / 2}} \mathrm{~d} a \mathrm{~d} \phi .
$$

This expression of the GHS measure is equivalent to those derived in (Carroll and Tam, 2010, Schiffrin and Wald 2012) 4

\footnotetext{
${ }^{4}$ There are some complications with the $k=1$ case. See (Schiffrin and Wald 2012 8) for the details. I have however chosen not to set $8 \pi G=1$, but rather maintained consistency with the rest of this dissertation's use of "geometrical units" by only setting $G=1$. Gibbons et al. (1987) use a simplifying, but less transparent coordinate choice. They also choose to investigate only the special case where $V=m^{2} \phi^{2} / 2$. It can be shown with some work that their expression is equivalent to this one as well with this potential.
} 


\section{The Flatness Problem}

The GHS measure clearly diverges for large scale factors, a point originally recognized by Gibbons et al. (1987. 745); it also converges to 0 for small scale factors. Due to the divergence, one may readily say that, given any choice of Hubble parameter $H_{*}$, almost all spacetimes will have a "large" scale factor. More precisely, pick any scale factor $a_{*}$; the set of spacetimes with $a<a_{*}$ is a negligible set: the total measure of this set is finite whereas the total measure of its complement is infinite. What is the significance of this fact about the GHS measure, specifically for the flatness problem?

Hawking and Page (1988, 803-4) suggest the following:

"Thus for arbitrarily large expansions (and long times), and for arbitrarily low values of the energy density, the canonical measure implies that almost all solutions of the Friedmann-RobertsonWalker scalar equations have negligible spatial curvature and hence behave as $k=0$ models. In this way a uniform probability distribution in the canonical measure would explain the flatness problem of cosmology...”

By "arbitrarily large expansions" (and "arbitrarily low values of energy density"), they appear to mean the following. Pick any arbitrary $a_{*}$ (and any arbitrary $\phi_{*}$ ) ${ }^{5}$ According to the GHS measure almost all spacetimes have $a>a_{*}$ (and $\phi>\phi_{*}$ ), or, equivalently, the spacetimes with $a<a_{*}$ (and $\phi<\phi_{*}$ ) compose a negligible set. Furthermore, since this holds for any choice of $a_{*}$, one may infer that almost all spacetimes are arbitrarily close to having $\kappa=0$ (since $\kappa=k / a^{2}$ ) in exactly the same sense. It is perhaps somewhat misleading to say that curved FRW spacetimes with large scale factors "behave as $k=0$ models;" the curvature does not change in such models. It is, however, surely false to say that a "uniform probability distribution" with respect to the GHS measure would explain the flatness problem of cosmology. There is in fact no such uniform probability distribution, since the GHS measure is not finite. Moroever, there is also no canonical probability distribution $\rho$ at all which would make $U \mapsto \int_{U} \rho \mathrm{d} \Omega_{G H S}$ into a probability measureone has to make a choice in order to obtain a probability measure in the case of infinite total measure, a choice which appears completely arbitrary in this context.

Carroll and Tam $(2010,14)$ invite us to consider the question in more "physically transparent" terms by looking at the curvature $\kappa$, which I previously exchanged in favor of the scale factor $a$ when deriving the GHS measure. One can recast the scale factor $a$ as the curvature $\kappa$ using the relation from before, namely $\kappa=k / a^{2}$. (Note especially that this switch maps the entire set of scale factors for the $k=0$ case to the single point $\kappa=0$.) One then defines the GHS measure (at least for curved FRW spacetimes) by the map

$$
U \mapsto \int_{U} \mathrm{~d} \Omega=-6 \int_{U} \frac{1}{|\kappa|^{5 / 2}} \frac{\left(3 H_{*}^{2}+2 \kappa\right) / 8 \pi-V}{\left((3 / 4 \pi)\left(H_{*}^{2}+\kappa\right)-2 V\right)^{1 / 2}} \mathrm{~d} \kappa \mathrm{d} \phi .
$$

It is clear that the measure diverges for small values of curvature, i.e. curvatures close to flat, due to the curvature term in the denominator. This is pointed out by Carroll and Tam (2010, 15). They suggest the following interpretation of this fact:

"Considering first the measure on purely Robertson-Walker cosmologies (without perturbations) as a function of spatial curvature, there is a divergence at zero curvature. In other words, curved [FRW] cosmologies are a set of measure zero-the flatness problem, as conventionally understood, does not exist."

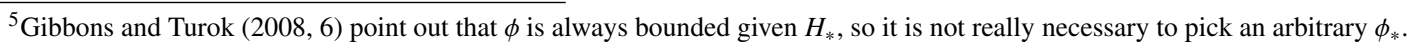


As stated these claims are highly suspect.

Firstly, Carroll and Tam assert that all values of their curvature coordinate $\Omega_{k}$ (essentially equivalent to $\kappa)$ can be integrated over. While this is perhaps true, portraying the phase space in terms of curvature is misleading. For curved FRW spacetimes, it is true that the measure diverges for small values of curvature $\kappa$, as I indicate above and as Hawking and Page suggest in the passage from their paper quoted above. The recast measure, however, is infinite at zero curvature because the entire set of $k=0$ scale factors is mapped to $\kappa=0$. The GHS measure diverges for large scale factors in the case of flat FRW spacetimes just as it does for curved FRW spacetime. Thus it is misleading to describe a "divergence at zero curvature;" there is nothing special going on in flat FRW spacetimes (at least in this respect) 6

Secondly (and relatedly), curved FRW spacetimes are clearly not a set of measure zero-at least according to the GHS measure. The initial data of FRW spacetimes is representable in the space $\{a, \dot{a}, \phi, \dot{\phi}, k\}$. The curvature constant $k$ serves as an index for three different phase spaces, each of which has an infinite total measure-even after taking into account constraints and choosing a hypersurface in the constraint surface according to GHS's procedure. The unboundedness of the total phase space measure for each kind of FRW spacetime is due, again, to the unbounded range of the scale factor Schiffrin and Wald $(2012,11) 7$ This is quite plain when one expresses the GHS measure in terms of the scale factor. Transforming to the curvature coordinate $\kappa$ should not change the fact that the total measure of each phase space is infinite. So, while it is true that the GHS measure attributes infinite measure to flat FRW spacetimes (as Carroll and Tam appear to recognize), it also does so both to positively curved FRW spacetimes and to negatively curved spacetimes. Therefore it is false that the curved FRW cosmologies are a set of measure zero according to the GHS measure; hence one cannot conclude on this basis that the flatness problem does not exist.

One might try to rescue Carroll and Tam's claim about the flatness problem by interpreting flatness more broadly, namely by including "nearly flat" curved spacetimes. This requires specifying what the set of "nearly flat" curved spacetimes is to be, e.g. a specification of the set of spacetimes with curvature less than some $\kappa_{*}$ (at some time corresponding to Hubble parameter $H_{*}$ ). Almost all spacetimes will have a "small" curvature $\kappa$ in comparison to this curvature $\kappa_{*}$. In other words, the set of spacetimes with $\kappa>\kappa_{*}$ is a negligible set. Since our universe's spatial curvature is thought to be "nearly flat," i.e. it should be less than $\kappa_{*}$ (whatever it is), it follows from this argument that our universe is actually typical, contra what is assumed in the flatness problem. Unfortunately this argument does not follow from the GHS measure alone, since one had to make an independent choice in choosing $\kappa_{*}$, a choice that is not natural in any clear sense whatever. Furthermore, it is doubtful that there is any reasonable argument to justify a choice of $\kappa_{*}$-an explication of "close to flat" in the context of FRW models; it appears to be a completely arbitrary choice.

Here is a slightly different tack into the same stiff headwind. Suppose $\kappa_{*}$ is the (non-zero) spatial curvature of our universe at the present time. The GHS measure can be used to infer that almost all spacetimes with the same Hubble parameter will have flatter spatial curvatures. In such circumstances, one might be inclined to wonder "Why is my universe's spatial curvature so large? It seems like it ought to be much smaller if my universe is typical!" On this line of thought, it seems like one actually has a curvature problem rather than a flatness problem. Of course one would say this for any $\kappa_{*}$ whatsoever, regardless of its magnitude,

\footnotetext{
${ }^{6}$ Carroll and Tam appear to equivocate several times between there being a divergence at $\kappa=0$ and the measure diverging as $\kappa \rightarrow 0$ : "The integral diverges near $[\kappa=0]$, which is certainly a physically allowed region of parameter space" (Carroll and Tam 2010, 17); "The measure diverges on flat universes" (Carroll and Tam 2010. 28).

${ }^{7}$ Besides in (Schiffrin and Wald 2012), this fact is correctly pointed out in (Gibbons et al. 1987. Hawking and Page 1988). While Carroll and Tam (2010 20-1) observe that "this divergence was noted in the original GHS paper, where it was attributed to 'universes with very large scale factors' due to a different choice of variables," they object to this as an interpretation: "This is not the most physically transparent characterization, as any open universe will eventually have a large scale factor." For this reason they exchange the scale factor for curvature; it is not clear, however, how this characterization is more physically transparent since it amounts to the same thing.
} 
so it is not clear how one would ever be in the position to be satisified with one's curvature in an FRW universe - at least insofar as one expects things in our universe to be typical (in accord with Copernican principle-style reasoning). No matter. The measure suggests this question. What is the answer?

The answer is that the curvature depends on the actual dynamical history of the universe, and so it has no explanation within the context of the HBB model (apart from one depending on an initial condition). That answer may be unsatisfying, but the question is a bad one anyway, driven by misleading intuitions. There is no such thing as a typical FRW spacetime, and the GHS measure is not going to explain why the universe's curvature is what it is. This kind of thinking is clearly motivated by supposing that the GHS measure can be used as a likelihood measure, as Carroll and Tam clearly do:

"When we consider questions of fine-tuning, however, we are comparing the real world to what we think a randomly-chosen history of the universe would be like" (Carroll and Tam, 2010, 11).

Some popular, specious conceptions (in physics and beyond) of statistical mechanics encourage this line of thought. Putatively successful typicality arguments in statistical mechanics (Goldstein, 2012) depend, however, not only on having a phase space measure, but also on both the dynamics of the system and on a specification of macroproperties or macrostates (defined as regions of phase space) (Frigg, 2009, Frigg and Werndl, 2012). Accordingly, any claim of fine-tuning in FRW spacetimes on the sole basis of the GHS measure (which does at least incorporate the FRW dynamics) is bound to miss the mark without additional assumptions (such as a well-motivated standard of flatness).

Gibbons and Turok (2008) take a different approach from Carroll and Tam. They correctly observe that universes with large scale factors are universes with small spatial curvatures. They then claim that the scale factor is neither "geometrically meaningful" nor "physically observable" and therefore propose to identify all the "indistinguishable" nearly flat spacetimes on the surface identified by $H_{*}{ }^{8}$ They do so by effectively choosing a "cutoff" curvature $\kappa_{*}$ and throwing out all the spacetimes with curvatures smaller than it. The advantage to doing this is that the total measure of FRW spacetimes with curvatures larger than $\kappa_{*}$ is finite, so that one can then define a probability measure in a natural way.

The disadvantage is that this makes no sense. Carroll and Tam (2010, 20) comment, "to us, this seems to be throwing away almost all the solutions, and keeping a set of measure zero. It is true that universes with almost identical values of the curvature parameter will be physically indistinguishable, but that doesn't affect the fact that almost all universes have this property." Indeed, doing what Gibbons and Turok do is throwing away almost all the solutions (although the remaining set has finite measure, not measure zero as Carroll and Tam claim). They are also right to point out that if nearly flat universes are physically indistinguishable, so are "nearly- $\kappa$ " universes for almost any $\kappa$. Gibbons and Turok do not throw out these universes however (else they would not have been left with any universes at all). Their justification for an additional assumption therefore fails.

Ironically, Carroll and Tam make essentially the same error as Gibbons and Turok, by identifying the flat and nearly flat spacetimes. Instead of throwing out all the flat and nearly flat spacetimes like the latter pair, however, the former pair throws out the complement of the flat and nearly flat spacetimes by assigning them zero measure. They then triumphantly conclude that all FRW spacetimes are essentially flat! Carroll and Tam propose to tame the remaining divergence in the GHS measure by regularizing the integral, in effect making the measure finite. The problem with doing this is that, since the GHS measure is not finite,

\footnotetext{
${ }^{8}$ It is not clear what they mean by "geometrically meaningful." The scale factor is clearly geometric in the relevant sense, since it relates spaceforms of the same kind by scalings. It is moreover physically meaningful because space is expanding (or contracting) in FRW spacetimes. The precise value of $a$ does not matter, as it can be re-scaled, but that does not undermine its meaningfulness. It is also unclear how the fact that $a$ is physically unobservable should matter, since most features of spacetime are not observable, e.g. the metric $g$, the spatial curvature $\kappa$, etc. The physically relevant content of these, including the scale factor, can be inferred from observations and appropriate assumptions.
} 
regularizing the measure makes it no longer the GHS measure, in which case any justification the measure had by its "naturalness" is lost since a choice was made ${ }^{9}$ In short, one may as well have just assumed the probability distribution they end up with from the very beginning.Their stated justification for this move is pragmatic: "This non-normalizability is problematic if we would like to interpret the measure as determining the relative fraction of universes with different physical properties" (Carroll and Tam, 2010, 17). However this is obviously an inadequate justification for the propriety of their measure.

\section{References}

Albrecht, Andreas, and Paul Steinhardt. "Cosmology for Grand Unified Theories with Radiatively Induced Symmetry Breaking." Physical Review Letters 48: (1982) 1220-1223.

Belinsky, Vladimir, Leonid Grishchuk, Isaak Khalatnikov, and Yakov Zeldovich. "Inflationary Stages in Cosmological Models with a Scalar Field.” Physics Letters B 155: (1985) 232-236.

Carroll, Sean. "In What Sense Is the Early Universe Fine-Tuned?" In Time's Arrows and the Probability Structure of the World, edited by Barry Loewer, Brad Weslake, and Eric Winsberg, Cambridge, MA: Harvard University Press, forthcoming.

Carroll, Sean, and Heywood Tam. "Unitary Evolution and Cosmological Fine-Tuning." ArXiv Eprint, 2010. http://arxiv.org/abs/1007.1417.

Coule, David. "Canonical measure and the flatness of a FRW universe." Classical and Quantum Gravity 12: (1995) 455-469.

Dicke, Robert, and Jim Peebles. "The Big Bang Cosmology_Enigmas and Nostrums." In General Relativity: An Einstein Centenary Survey, edited by Stephen Hawking, and Werner Israel, Cambridge: Cambridge University Press, 1979, chapter 9, 504-517.

Frigg, Roman. "Typicality and the Approach to Equilibrium in Boltzmannian Statistical Mechanics." Philosophy of Science 76: (2009) 997-1008.

Frigg, Roman, and Charlotte Werndl. "Demystifying Typicality.” Philosophy of Science 79: (2012) 917-929.

Geroch, Robert. "General Relativity.”, 1972. Unpublished lecture notes.

Gibbons, Gary, Stephen Hawking, and John Stewart. "A natural measure on the Set of all Universes." Nuclear Physics B 281: (1987) 736-751.

Gibbons, Gary, and Neil Turok. "Measure problem in cosmology." Physical Review D 77: (2008) 1-12.

Goldstein, Sheldon. "Typicality and Notions of Probability in Physics." In Probability in Physics, edited by Yemima Ben-Menahem, and Meir Hemmo, Berlin: Springer Verlag, 2012, chapter 4, 59-71.

Guth, Alan. "Inflationary universe: A possible solution to the horizon and flatness problems." Physical Review D 23, 2: (1981) 347-356.

\footnotetext{
${ }^{9}$ Carroll more recently has conceded the artificiality of regularizing: "Earlier attempts to regularize the measure, for example by considering an $\epsilon$-neighborhood around the zero-curvature Hamiltonian constraint surface (Carroll and Tam 2010) or by identifying universes with similar curvatures (Gibbons and Turok 2008) have not proven satisfactory" (Remmen and Carroll|2013 7). He remains convinced, however, that almost all FRW spacetimes are "nearly flat:" "we should throw all of the others away and deal with flat \begin{tabular}{l|l|l||l|l|} 
universes," Carroll & forthcoming 19), developing a measure on just these spacetimes in a later paper (Remmen and Carroll & 2014
\end{tabular}
} 
Hawking, Stephen, and Don Page. “How Probable is Inflation?” Nuclear Physics B 298: (1988) 789-809.

Linde, Andrei. "A New Inflationary Universe Scenario: A Possible Solution of the Horizon, Flatness, Homogeneity, Isotropy, and Primordial Monopole Problems." Physics Letters B 108: (1982) 389-393.

__. "The inflationary universe." Reports on Progress in Phyics 47: (1984) 925-986.

Malament, David. Topics in the Foundations of General Relativity and Newtonian Gravity Theory. Chicago: University of Chicago Press, 2012.

McCabe, Gordon. "The structure and interpretation of cosmology: Part I-general relativistic cosmology." Studies in History and Philosophy of Modern Physics 35: (2004) 549-595.

McCoy, Casey. "Does inflation solve the hot big bang model's fine-tuning problems?" Studies in History and Philosophy of Modern Physics 51: (2015) 23-36.

Remmen, Grant, and Sean Carroll. "Attractor solutions in scalar-field cosmology." Physical Review D 88: (2013) $1-14$.

$1-14$.

Schiffrin, Joshua, and Robert Wald. "Measure and probability in cosmology." Physical Review D 86: (2012) $1-20$.

Smeenk, Chris. "Philosophy of Cosmology." In The Oxford Handbook of Philosophy of Physics, edited by Robert Batterman, Oxford: Oxford University Press, 2013, chapter 17, 607-652.

Wald, Robert. General Relativity. Chicago: University of Chicago Press, 1984.

Wolf, Joseph. Spaces of Constant Curvature. Providence, RI: AMS Chelsea Publishing, 2010, 6th edition. 